\title{
EL SISTEMA ELECTORAL ITALIANO: LO QUE EL LEGISLADOR HACE, EL TRIBUNAL LO DESHACE
}

MARCO CECILI 
SUMARIO

1. PREMISA - 2. DESDE 2005 HASTA 2014: TRES ELECCIONES CON UNA LEY ELECTORAL INCONSTITUCIONAL - 3. UNA NUEVA LEY ELECTORAL NUNCA EMPLEADA, PERO INCONSTITUCIONAL - 4. UNA LEY ELECTORAL APROBADA (AL MARGEN DE LAS ELECCIONES) QUE DESCONTENTA A TODOS. - 5. LAS ELECCIONES DEL 4 DE MARZO DE 2018 Y EL ESTANCAMIENTO EN LA FORMACIÓN DEL GOBIERNO - 6. UN SISTEMA EN LA ANSIADA BÚSQUEDA DE UN EQUILIBRIO. 


\title{
EL SISTEMA ELECTORAL ITALIANO: LO QUE EL LEGISLADOR HACE, EL TRIBUNAL LO DESHACE
}

\author{
MARCO CECILI \\ Estudiante de doctorado en Derecho Público \\ Universidad de Roma Tor Vergata ${ }^{1}$
}

\section{PREMISA}

En los últimos años, el sistema electoral italiano ha sufrido una cantidad de reformas de notable interés. Desde 2014, tanto el Tribunal Constitucional como el legislador han sido protagonistas de un diálogo, quizás único en el mundo, que todavía parece no haber terminado ${ }^{2}$. Desde 2005 hasta hoy, el legislador italiano ha aprobado tres leyes electorales, dos de las cuales han sido declaradas parcialmente inconstitucionales por el Tribunal Constitucional. En noviembre de 2017, en el la Gazzetta Ufficiale fue publicada una nueva ley electoral (ley 165/2017) tanto para la Cámara de Diputados como para el Senado. El nuevo sistema puede ser clasificado como "mixto" y contempla elementos tanto proporcionales como mayoritarios. El propósito de este texto es analizar el particular caso italiano desarrollado en los últimos cuatro años, así como la gestación y el nacimiento de la nueva normativa electoral, la llamada "Ley Rosato" (denominada así por el diputado Ettore Rosato, relator de la misma). En las últimas elecciones generales italianas, que se celebraron el 4 de marzo de 2018, se votó ya bajo el marco del nuevo sistema electoral (implementado por la

${ }^{1}$ Universidad de Roma Tor Vergata. Dipartimento di Giurisprudenza. Via Cracovia, n. 50. 00133 Roma. Email: marcocecili@gmail.com

Mis más sinceros agradecimientos a los doctores Sara Ramos Romero (Universitat Pompeu Fabra de Barcelona), Roberto Carbone (Universitad de Roma Tor Vergata), Giuliano Vosa (Centro de Estudios Políticos y Constitucionales / Universitad LUISS de Roma) y a Valentina Maggi por la ayuda en la redacción del texto, a los profesores Giuseppe Martinico (Escuela Superior Sant'Anna de Pisa) y Matteo Nicolini (Universidad de Verona) por sus valiosas sugerencias.

2 Baraggia, A. (2017). "Italian Electoral Law: A Story of an Impossible Transition?". Election Law Journal, 2, pp. 272-279.

${ }^{3}$ Sgrò, F. (2017). "Prime considerazioni sulla legge n. 165 del 2017: questioni nodali e specificità del nuovo sistema elettorale italiano". Osservatorio AIC, 3, p. 2. 
Ley Rosato). El resultado ha llevado a la constitución de un Parlamento dividido en tres grandes bloques que ha creado un estancamiento irresoluble entre las fuerzas políticas que, de nuevo, pretenden la enésima modificación de las reglas electorales

\section{DESDE 2005 HASTA 2014: TRES ELECCIONES CON UNA LEY ELECTORAL INCONSTITUCIONAL}

No se puede resumir la abundante literatura que se ha ocupado de la ley electoral 270/2005, pero parece ser conveniente resumir su historia ${ }^{4}$.

En 2005, al final de la legislatura XIV, la mayoría parlamentaria de centroderecha aprobó una reforma electoral (llamada "ley Mattarella" de 1993) que puso término al sistema anterior basado principalmente en las circunscripciones uninominales, con una rectificación proporcional. La ley 270/2005 (conocida como ley Porcellum 5 , denominación que le dio el ponente Calderoli después de varias modificaciones) con-

${ }^{4}$ Para un examen más detallado de la decisión del Tribunal Constitucional n. 1/2014 véase la charla entre los autores en Jurisprudencia constitucional (2014) y en Nomos. Le attualità del diritto, entrega n. 3, 2013. Véase también Rauti, A. (2014), I sistemi elettorali dopo la sentenza costituzionale n. 1 del 2014. Problemi eprospetti, Nápoles, Editoriale Scientifica Italiana; D’Amico, M. y Catalano, S. (2014), Prime riflessioni sulla "storica" sentenza 1 del 2014 in materia elettorale, Milán, FrancoAngeli. Para una reflexión sobre la ley 270/2005 y la representación, véase De Fiores, C. (2009). Rappresentanza politica e legge elettorale, Túrin, Giappicchelli. Entre los comentarios de la decisión 1/2014, véanse Dickmann, R. (2014). "La Corte dichiara incostituzionale il premio di maggioranza e il voto di lista e introduce un sistema elettorale proporzionale puro fondato su una preferenza". Federalismi, 2; Caravita, B. (2014). "La riforma elettorale alla luce della sent. 1/2014". Federalismi, 2; Nicotra, I. (2014). "Proposte per una nuova legge elettorale alla luce delle motivazioni contenute nella sentenza della Corte costituzionale n. 1 del 2014". Consulta Online; Spadacini, L. (2014). "I limiti alla discrezionalità del legislatore in materia elettorale desumibili dalla sentenza della Corte costituzionale n. 1 del 2014”. Forum di Quaderni Costituzionali, 1; Pertici, A. (2014). "La Corte costituzionale dichiara l'incostituzionalità della legge elettorale tra attese e sorprese (con qualche indicazione per il legislatore)". Forum di Quaderni Costituzionali, 1; Trucco, L. (2014). "Il sistema elettorale Italicum alla prova della sentenza della Corte costituzionale n. 1 del 2014 (note a prima lettura)". Consulta Online; Pesole, L. (2014). "L'incostituzionalità della legge elettorale nella prospettiva della Corte costituzionale, tra circostanze contingenti e tecniche giurisprudenziali già sperimentate". Costituzionalismo, 2; Caredda, M. (2014). "La retroattività bilanciabile. Ragionando intorno alla sentenza n. 1 del 2014 della Corte costituzionale”. Costituzionalismo, 2.

Sobre las novedades de la decisión con referencia a las características del derecho de voto y a la representancia política, véanse, entre los otros, Armanno, M. (2014). "Diritto di voto, rappresentanza ed evoluzione del sistema dei partiti politici. Riflessioni a margine della recente giurisprudenza costituzionale". Rivista AIC, 4; Lehner, E. (2014). "Il diritto di voto dopo la conquista della "zona franca", Giurisprudenza costituzionale, 1, pp. 54-63; Rescigno, G.U. (2014), "Il diritto costituzionale di voto secondo la Corte di cassazione e la Corte costituzionale”. Giurisprudenza costituzionale, 1, p. 27; Rivosecchi, G. (2016). "I partiti politici nella giurisprudenza costituzionale". Osservatorio costituzionale, 3, p. 13.

5 El relator la definió "porcata", palabra que se puede traducir con "guarrada". 
templaba un sistema proporcional, pero caracterizado por la presencia de un premio de mayoría ${ }^{6}$.

En la elección de la Cámara de Diputados, se contemplaba primero un premio de mayoría flexible (majority assuring) para la lista o la coalición que hubiera obtenido más votos con el fin de garantizar de esta manera al menos 340 escaños (el $54 \%$ de la Cámara $)^{7}$. Mientras, los escaños restantes se distribuían proporcionalmente entre las otras listas y coaliciones. En segundo lugar, se preveían varios umbrales mínimos de votos para participar en las reparticiones de los escaños, esto es: el 10\% de votos para las coaliciones, aunque participaban en esta repartición sólo las listas que superaban el 2\% (además, había la repesca del primer partido por debajo del 2\%, "clausola del miglior perdente" ${ }^{8}$ ), y el $4 \%$ de votos para las listas no coligadas. Por último, se adoptaban listas cerradas y bloqueadas, es decir, listas de candidatos cuyo orden no podía ser modificado por parte del elector, que, a su vez, no podía expresar una verdadera preferencia.

Para ganar y obtener el premio de mayoría de 340 escaños era necesario recibir un voto más con respecto a la lista o a la coalición adversaria.

En cambio, este sistema electoral preveía la distribución de escaños a nivel regional para el Senado. Se atribuían los $299^{9}$ escaños del Senado previendo 17 premios de mayoría regionales. De tal modo que, a quien obtenía más votos en una región, se le asignaba el $55 \%$ de los escaños que habían sido concedidos a esa región; mientras que el restante $45 \%$ de escaños, se redistribuía de manera proporcional. Además, los umbrales mínimos estaban calculados sobre base regional con un $20 \%$ para las coaliciones (participaban en la redistribución las listas coligadas que obtenían al menos el $3 \%$ de votos) y un $8 \%$ para las listas no coligadas.

El sistema electoral del Senado se configuraba como una verdadera "lotería" Debido a los diecisiete diferentes premios regionales, no sólo no había garantías de una mayoría sólida, ni siquiera se aseguraba que la mayoría de los escaños fuesen ganados por la coalición que había obtenido más votos con respecto a las otras y, tampoco, que fuesen ganados por la misma coalición que había ganado en el Congreso de los Diputados ${ }^{11}$.

${ }^{6}$ Fusaro, C. (2009). "Party System Developments and Electoral Legislation in Italy (1948-2009)". Bulletin of Italian Politics, 1, pp. 57-58.

${ }^{7}$ Por supuesto, este sistema no encontraba aplicación si la lista o la coalición obtenía ya autónomamente más del $54 \%$ de escaños.

8 «Cláusula del mejor perdedor». En la suma de los votos se cuentan también los votos de las listas que no alcanzan el umbral mínimo.

9 Hecha excepción de los 6 escaños destinados a los italianos residentes en el extranjero, de los 7 para Trentino- Alto Adigio, de los dos para Molise y del único escaño para Valle De Aosta.

${ }^{10}$ D'Alimonte, R (2014)., "Il Senato: una lotteria senza vincitori”, en Chiaramonte A. y De Sio L., Terremoto elettorale, Le elezioni politiche del 2013, Bolonia, Il Mulino, pp. 153-172.

${ }_{11}$ No hay que olvidar que vota para el Senado quien ha cumplido los 25 años, mientras que para votar para la Cámara es necesario ser mayor de 18 años. 
El sistema, criticado duramente por la opinión pública y por las fuerzas políti$\operatorname{cas}^{12}$, fue declarado parcialmente inconstitucional con la decisión del Tribunal Constitucional $1 / 2014^{13}$.

El Tribunal, superando definitivamente una corriente doctrinal y jurisprudencial que consideraba incuestionables las leyes electorales, decidió declarar procedente el recurso ${ }^{14}$.

Por lo que concierne a los premios de mayoría, el Tribunal examinó el perfil lògico del premio de mayoría en la economía del sistema electoral ${ }^{15}$ verificando el balance de los intereses constitucionalmente relevantes en juego, en vista de una "prueba de proporcionalidad". El objetivo era comprobar si las normas escrutadas llevarán, entre las posibles soluciones a la luz de los objetivos que se debían alcanzar, a la solución «menos restrictiva de los derechos comparados» y a las «cargas no desproporcionadas en relación con la persecución de dichos objetivos».

En cuanto al premio de mayoría, la legislación analizada pretendía facilitar la formación de una adecuada mayoría parlamentaria que garantizara la estabilidad de gobierno, intento que el Tribunal consideraba constitucionalmente legítimo, pero tenía que ser perseguido con correctivos para que decayera lo que el Tribunal llamaba ratio de la fórmula electoral, es decir, asegurar la representatividad del Parlamento. La disciplina electoral no era proporcionada al objetivo perseguido, puesto que provocaba una excesiva limitación de la función representativa parlamentaria y, también, del igual derecho de voto, contribuyendo a producir una alteración profunda de la composición representativa. Citando el Bundesverfassungsgericht $t^{16}$, la decisión enfatizaba este elemento, subrayando que:

En caso de que el legislador adopte el sistema proporcional, aunque de manera parcial, esto provocaría en el elector la legítima esperanza de que no se vaya a determinar un desequilibrio en los efectos del voto, una desigual evaluación del peso del

12 Esta ley electoral fue empleada en 2006, 2008 y 2013. Incluso el mismo Tribunal Constitucional hizo una amonestación sobre la constitucionalidad de la ley 270/2005 en las decisiónes 15, 16 y 17 de 2008.

13 En 2009, la ley 270/2005 fue sometida (bajo algunos aspectos, entre los cuales la posibilidad de presentar coaliciones) a referendos abrogatorios, pero este automatismo no surtió efectos por la falta de obtención del quórum constitutivo. Véase Cerrina Feroni, G. (2009). "Il referendum elettorale del 21-22 giugno 2009: tra uso politico dell'astensione, prospettive di riforme e nostalgie proporzionalistiche". Federalismi, 13; Vuolo, A. (2017). La legge elettorale, Nápoles, Editoriale Scientifica Italiana, pp. 149-158.

14 Bin, R. (2014). “'Zone franche' e legittimazione della Corte”. Forum di Quaderni Costituzionali, 5 de mayo; Sobrino, G. (2017). Il problema dell'ammissibilità delle questioni di legittimità costituzionale della legge elettorale alla luce delle sentenze n. 1/2014 e n. 35/2017 e le sue possibili ricadute: dalla (non più tollerabile) "zona franca" alla (auspicabile) "zona a statuto speciale" della giustizia costituzionale?, Federalismi, 15 .

15 Marini, F. S. (2014). "La ragionevolezza come parametro incerto della costituzionalità delle leggi elettorali”, en Confronti costituzionali.

16 Para una comparación de la jurisprudencia italo-alemana, Delledonne, G. (2014). "Corti costituzionali e legislazione elettorale: una comparazione italo-tedesca". Rivista Trimestrale di diritto pubblico, 4, pp. 1031-1062. 
voto saliente, a efectos de la atribución de los escaños, aunque no sea necesaria para evitar un prejuicio respecto a la funcionalidad del órgano parlamentario ${ }^{17}$.

Esta afirmación tendría que empujar a prestar atención a los sistemas de base proporcional que tienen, sin embargo, excesivos mecanismos distorsionadores, sobre todo a la hora de alcanzar la mayoría de escaños porque menoscaban la expectativa producida en los electores debido a la elección legislativa de un sistema "de base" proporcional.

Además, el premio de mayoría previsto fue considerado inconstitucional. El sistema «no impone un umbral mínimo de votos a la lista (o coalición de listas) de mayoría relativa de votos [...], confiere automáticamente un número incluso más elevado de escaños que convierte una formación que ha perseguido un porcentaje muy reducido de sufragios en la que alcanza la mayoría absoluta de los diputados de la Cámara».

Finalmente, se desprende que el Tribunal no declaró inconstitucional el premio de mayoría en sí mismo, lo hizo sólo en la medida en que la ley no había establecido un correctivo que sirviese para garantizar una real representatividad del Parlamento basándose en las indicaciones del electorado, ni la consecución de un umbral mínimo de votos relacionado con la lista o a la coalición de mayoría relativa ${ }^{18}$.

En cuanto al Senado, el Tribunal constitucional declaró la inconstitucionalidad de otra parte de la Ley, según la cual el premio de mayoría del Senado se calculaba en base regional. El Tribunal planteaba que, en consecuencia de la aplicación de los premios, obtener una real mayoría en el Senado correspondía al «resultado casual de una suma de premios regionales».

Ambos sistemas fueron declarados inconstitucionales también por lo que concierne el aspecto de las "listas bloqueadas". De hecho, según el Tribunal «es la circunstancia que a la totalidad de los parlamentarios elegidos, sin alguna excepción, falta el apoyo de la indicación personal por parte de los ciudadanos, que hiere la lógica de la representación entregada por la Constitución», en contradicción con el principio democrático, que se basa en la libertad de voto contemplada en el artículo 48 de la Constitución italiana. El Tribunal censuró que el sistema electoral excluía «cada autoridad del elector de influir en la elección de sus propios representantes» porque el orden de presentación estaba decidido por los partidos y no era de ninguna manera modificable por los electores ${ }^{19}$.

${ }^{17}$ [En adelante, la traducion es propria]. Véase Tribunal Constitucional, decisión del 13 de enero de 2014, n. 1. La jurisprudencia mencionada es BVerfGE, decisión 3/11 del 25 de julio de 2012 (2 BvF 3/11, 2 BvR 2670/11, 2 BvE 9/11). Véanse asimismo la decisión n. 197 del 22 de mayo de 1979 (BVerfGE 51, 222) y la decisión n. 1 del 5 de abril de 1952 (2 BvH 1/52).

${ }_{18}$ Guzzetta, G. (2014). "La sentenza 1 del 2014 sulla legge elettorale a una prima lettura”. Forum di Quaderni Costituzionali, 14 de enero.

19 Scaccia, G. (2014). "Riflessi ordinamentali dell'annullamento della legge n. 270 del 2005 e riforma della legge elettorale”. Forum di Quaderni Costituzionali, 14 de enero, pp. 7-8. 
Semejantes condiciones de voto imponían al ciudadano, seleccionando una lista, que eligiera en bloque incluso todos los numerosos candidatos en ella listados, que no había tenido la oportunidad de conocer y evaluar y que, de forma automática, estaban destinados a convertirse en diputados o senadores, según su posición en la lista. Esto hacía que la disciplina no pudiera ser comparada con otros sistemas caracterizados por listas bloqueadas sólo por una parte de escaños, ni con otros caracterizados por circunscripciones electorales de dimensiones territorialmente reducidas, en las que el número de los candidatos que debían ser elegidos era tan pequeño que permitía que se conociera de antemano y que la elección y la libertad de voto fueran efectivas. El derecho de la ciudadanía a la elección del órgano legislativo, que se desprende de la lectura conjunta A de los artículos 48, 49, 56, 58, 67 de la Constitución italiana y del artículo 3 del Protocolo 1 del CEDH (Convenio Europeo de Derechos Humanos) ${ }^{20}$, implica la facultad del elector de influir en la elección de sus propios representantes y, por lo tanto, no es compatible con mecanismos de voto que remiten totalmente esta elección a los partidos políticos, a través del mecanismo de las listas bloqueadas. La elección en favor de la lista bloqueada no parece ser excluida en principio, pero sí cuando la lista sea tan "extensa" como para impedir al ciudadano conocer y evaluar los numerosos candidatos en ella aparecen.

Teniendo en cuenta lo que se ha dicho, el Tribunal declaró la inconstitucionalidad en primer lugar de la falta de predicción de cualquier umbral de acceso al premio de mayoría nacional para la Cámara de Diputados, porque contrariaba la ratio de la fórmula electoral proporcional, provocando una excesiva diferencia entre la composición del órgano de representación política y la voluntad de los ciudadanos expresada a través del voto. Además, reputó inconstitucional el mecanismo de asignación del premio de mayoría a escala regional para el Senado, porque reprimía la representatividad del sistema de manera desproporcionada con respecto del objetivo perseguido de garantizar la estabilidad de gobierno y remitía la total composición del premio a elementos casuales que pueden cambiar el resultado obtenido por las listas a base nacional y, por lo tanto, era contradictorio respecto a la misma lógica de tutela de la gobernabilidad. Por último, fue declarado inconstitucional el voto basado en las listas bloqueadas porque aplicado a la totalidad de los parlamentarios elegidos, impedía al

20 El TEDH ya analizó la ley 270/2005 en la decisión Saccomanno vs. Italia del 13 de marzo de 2012. Los jueces de Estrasburgo consideraron las listas bloqueadas coherentes con el sistema TEDH, «el legislador ha establecido unos mecanismos de este tipo, en particular con el fin de combatir la influencia del crimen organizado en el resultado electoral, habida cuenta del contexto específico italiano, prevenir el comercio de los votos y favorecer la emergencia de candidatos que poseen competencias específicas». El Tribunal se ha ocupado también de una disciplina comparada, visto que los sistemas electorales europeos no desconocen la problemática de las listas bloqueadas y/o cerradas. A este respecto, del análisis de los Estados miembros con un sistema electoral similar al italiano, se desprende que la mayoría de éstos ha adoptado un sistema que prevé listas de candidatos bloqueadas, al menos en una de las cámaras legislativas, o sea, 13 Estados miembros de un total de 22. Véase Ferrari, F. (2014). "Liste bloccate o situazione normativa? Un'alternativa all'oggetto del giudizio di costituzionalità”. Forum di Quaderni Costituzionali, 21 de enero. 
ciudadano evaluar a los candidatos listados y ello hacía que a la elección le faltara la indicación personal de los ciudadanos, agraviando así la lógica de la representación política.

Después de la decisión del Tribunal, los sistemas llegaron a ser proporcionales (puesto que el premio de mayoría había sido declarado inconstitucional), con umbrales mínimos ${ }^{21}$ de voto y, tras la eliminación de las listas bloqueadas, se permitió al elector, de facto, la posibilidad de expresar, al menos, una preferencia.

\section{UNA NUEVA LEY ELECTORAL NUNCA EMPLEADA, PERO INCONSTITUCIONAL}

Bajo el impulso del entonces presidente del Gobierno Matteo Renzi, en mayo de 2015, se aprobó una nueva ley electoral para la Cámara de Diputados, (conocida como (talicum $)^{22}$.

El sistema electoral resultante era de base proporcional, pero con la presencia de un premio de mayoría de 340 escaños ( $55 \%$ de los escaños) a la lista (no a la coalición) que hubiera alcanzado al menos el $40 \%$ de votos ${ }^{23}$.

En caso de que ninguna fuerza política hubiera alcanzado este porcentaje de votos, habría sido necesario realizar una segunda votación entre las dos listas más votadas. Quien hubiera obtenido más votos, habría obtenido el $55 \%$ de los escaños, mientras que, al vencido, se habrían asignado los escaños siguiendo el resultado de la primera votación.

El sistema preveía un único umbral mínimo de votos del 3\% a nivel nacional para todas las listas.

Para la designación de los votados, el territorio nacional había sido dividido en 100 circunscripciones plurinominales (designadas posteriormente con un decreto legislativo 189 de 2017) en las que estaba presente un cabeza de lista "bloqueado" y otros candidatos que debían ser elegidos a través de preferencias.

21 No se planteó el problema de constitucionalidad a propósito de los umbrales mínimos de votos.

22 Ley del 6 de mayo de 2015, n. 52. Para conocer los primeros comentarios sobre el Italicum, véase Canale, G. (2015). "Italicum: Itaca, sognando la California (una prima lettura critica della legge n. 52/2015)". Consulta Online, 2, pp. 437-448; Fusaro, C. (2015). "Le critiche al ballottaggio dell'Italicum o del rifiuto di rafforzare la governabilità pervia elettorale attraverso il premio alla lista". Forum di Quaderni Costituzionali, 23 de junio; Lanchester, F. (2015). "Il sistema elettorale in senso stretto dal Porcellum all'Italicum". Democrazia e diritto, 1, pp. 15-29; Mostacci, E. (2015). "Perseverare autem italicum: rappresentanza e sistema politico all'indomani della legge 52/2015”. Rivista AIC, 4, 2015; Rauti, A. (2015). "L'italicum fra 'liturgia' elettorale e prove di bipartitismo". Rivista AIC, 2; Scaccia, G. (2015). "La legge elettorale 'Italicum' fra vincoli sistemici ed equilibri costituzionali”. Questione Giustizia, 1, pp. 16-23.

23 D'Alimonte, R. (2015). "The new Italian electoral system: majority-assuring but minorityfriendly”. Contemporary Italian Politics, 3, pp. 286-292. 
Además, dada la fuerte conexión con la ley de revisión constitucional propuesta por el presidente Renzi (que habría convertido la elección del Senado en elección indirecta por parte de los Consejos regionales), no se había previsto una disciplina electoral para la segunda Cámara. Por esta razón, la entrada en vigor de la ley se pospuso al 1 de julio de 2016 (fecha dentro de la que el gobierno preveía que la reforma de la Constitución, que se estaba debatiendo en Parlamento, habría terminado su tramitación y el Senado ya no habría sido elegido de forma directa). Sin embargo, la reforma constitucional, votada con carácter definitivo por el Parlamento italiano, no fue aprobada en el sucesivo referéndum constitucional del 4 de diciembre de $2016^{24}$.

Con la decisión 35 de $2017^{25}$, el Tribunal Constitucional italiano declaró parcialmente inconstitucional esta ley electoral ${ }^{26}$.

En primer lugar, el Tribunal afirmó que:

«el legislador puede introducir un premio de mayoría en un sistema electoral que se inspira en el criterio del reparto proporcional de escaños, siempre que este

${ }^{24}$ Para un ahondamiento de las mayores novedades que la reforma habría conllevado véase Marini, F. S. y Scaccia, G. (2016). Commentario alla Riforma costituzionale del 2016, Nápoles, Editoriale Scientifica italiana.

25 Para una lectura de la decisión 35/2017, véase Ceccanti, S. (2017). "I sistemi elettorali per le elezioni politiche dopo la 35/2017: una sentenza figlia del referendum, ma per il resto deludente per i proporzionalisti”. Federalismi, 4; Dickmann, R.(2017). "La Corte costituzionale trasforma l'Italicum in sistema elettorale maggioritario 'eventuale' ma lascia al legislatore l'onere di definire una legislazione elettorale omogenea per le due Camere". Federalismi, 4; Morrone, A. (2017). "Dopo la decisione sull'Italicum: il maggioritario è salvo, e la proporzionale non un obbligo costituzionale”. Forum di Quaderni Costituzionali, 13 de febrero; Ruggeri, A. (2017). "La Corte alla sofferta ricerca di un accettabile equilibrio tra le ragioni della rappresentanza e quelle della governabilità: un'autentica quadratura del cerchio, riuscita però solo a metà, nella pronunzia sull'Italicum”. Forum di Quaderni Costituzionali, 25 de febrero; Trucco, L. (2017). “'Sentenza Italicum': la Consulta tra detto, non considerato e lasciato intendere”. Consulta Online, 1; Giupponi, T. F. (2017). “"Ragionevolezza elettorale» e discrezionalità del legislatore, tra eguaglianza del voto e art. 66 Cost.”. Forum di Quaderni Costituzionali, 18 de junio; Comazzetto, G. (2017). "Fictio litis e azioni di accertamento del diritto costituzionale di voto dopo la sentenza 35/2017". Forum di Quaderni Costituzionali, 21 de junio de 2017; Massa Pinto I. (2017). "Dalla sentenza n.1 del 2014 alla sentenza n. 35 del 2017 della Corte costituzionale sulla legge elettorale: una soluzione di continuità c'è e riguarda il ruolo dei partiti politici”. Costituzionalismo, 1; Rauti, A. (2017). "La Corte costituzionale ed il legislatore. Il caso emblematico del controllo di costituzionalità sulle leggi elettorali", en Butturini, D. y Nicolini M., Giurisdizione costituzionale e potere democraticamente legittimato, Vol I, Bolonia, Bononia University Press, pp. 171-222.

26 Esposito, M. (2017). "Diritto di voto e sistema elettorale: riflessioni a margine della sent. n. 35/2017 della Corte costituzionale”. Giurisprudenza costituzionale, 1, pp. 549-566; Luciani M. (2017). "Bis in idem: la nuova sentenza della Corte costituzionale sulla legge elettorale politica". Rivista AIC, 1; Delledonne, G. y Boggero, G. (2017). "The Italian Constitutional Court Rules on Electoral System”, en http://www.iconnectblog.com/2017/02/the-italianconstitutional-court-rules-on-electoral-system; Delledonne, G.. (2017). "Legge elettorale e principi costituzionali in materia di partiti politici: un'interazione problematica”. Quaderni costituzionali, 4, pp. 801-823; Faraguna P. (2017). "Do You Ever Have One of Those Days When Everything Seems Unconstitutional? The Italian Constitutional Court Strikes Down the Electoral Law Once Again: Italian Constitutional Court Judgment of 9 February 2017 No. 35". European Costitutional Law Review, 4, pp. 778-792. 
mecanismo de premios no anuncie una excesiva sobrerrepresentación de la lista de mayoría relativa ${ }^{27}{ }$.

Y, además, subrayó:

«El legislador consideró necesario cumplir con las indicaciones de la jurisprudencia constitucional, tanto previendo un umbral mínimo de votos para la asignación del premio de mayoría, como estableciendo que, en caso de que ninguna lista alcance 340 escaños, se proceda a un segundo escrutinio entre las dos listas más votadas. Si la primera predicción no determina una irrazonable compresión de la representatividad del organismo electivo son, en cambio, las concretas modalidades de asignación del premio a través del segundo escrutinio las que provocan la violación de los artículos 1, párrafo segundo, 3 y 48, párrafo segundo de la Constitución».

Según el Tribunal, en el sistema esbozado en la ley 52 de 2015, la segunda ronda de votos no había sido ideado como una nueva votación con respecto a la realizada en la primera ronda, sino como su prosecución. En esta perspectiva, al segundo voto accedían las únicas dos listas más votadas en la primera ronda, sin que fueran permitidas, en las dos vueltas, formas de conexión o alianza política entre listas. Además, incluso después de la realización del segundo escrutinio, la repartición porcentual de escaños seguía siendo (para todas las listas diferentes de la ganadora, y también, como se ha dicho anteriormente, para la que participaba en el segundo escrutinio perdiendo) la misma de la primera vuelta. La segunda votación servía, entonces, «para identificar la lista ganadora, o sea para permitir a una lista que alcanzara ese umbral mínimo de votos que ninguna había obtenido en la primera vuelta».

Pero el Tribunal se dio cuenta de que una lista habría podido acceder a la segunda ronda electoral incluso habiendo obtenido, en la primera, un consenso exiguo, y, no obstante eso, habría podido ganar potencialmente el premio y habría conseguido más del doble los escaños que habría podido obtener basándose en los votos recibidos en la primera vuelta. De esta manera, este mecanismo reproducía, si bien en la segunda ronda, un efecto distorsionador análogo a lo que esto Tribunal identificó en la decisión n. 1 de 2014, con relación a la legislación electoral anterior ${ }^{28}$.

El Tribunal, una vez más, declaró constitucional el objetivo de la estabilidad de Gobierno, de interés constitucional indudable. Pero éste no podía provocar un excesivo sacrificio de los dos principios constitucionales de la representatividad e igualdad del voto.

Como subrayó el Tribunal, era cierto que, frente al resultado del segundo escrutinio, el premio no se determinaba artificialmente, aunque procediera de un voto de los electores. Pero, si en la primera vuelta hubiera probado que ninguna lista hubiera sido capaz a solas de ganar el premio de mayoría, sólo las estrictas condiciones de

27 Tribunal Constitucional, decisión del 9 de febrero de 2017 , n. 35.

28 Casanova, D. y Spadacini, L. (2017). "Il ballottaggio nazionale tra liste: la sentenza Corte cost. n. 35 del 2017 e il 'de profundis' per i sistemi 'majority assuring'”. Osservatorio AIC, 2, pp. 7-8. 
acceso al segundo escrutinio habrían podido llevar a la segura asignación de este premio, gracias a una reducción drástica de la oferta política ${ }^{29}$.

Además, el Tribunal declaró inconstitucional un detalle relacionado con las pluricandidaturas. La ley preveía que el diputado elegido en más circunscripciones plurinominales tuviera que declarar a la Presidencia de la Cámara de Diputados, dentro de ocho días a partir de la fecha de la última proclamación, qué circunscripción plurinominal hubiera escogido.

Esta disposición permitía al cabeza de lista, elegido en más circunscripciones plurinominales, optar, basándose en su simple evaluación de oportunidades, no subordinada a algún criterio objetivo y predeterminado y respetuoso al máximo de la voluntad expresada por los electores. El plurielegido podía influir de manera arbitraria en la composición de las Càmaras. El Tribunal aconsejó emplear unos criterios objetivos (por ejemplo, donde el partido hubiese obtenido el mayor número de votos), pero en caso de especialidades (por la "normativa resultante del efecto abrogatorio") puso en práctica el sorteo ${ }^{30}$.

\section{UNA LEY ELECTORAL APROBADA AL MARGEN DE LAS ELECCIONES QUE DESCONTENTA A TODOS.}

Después de la llamada sentenza 35 en Italia hubo dos sistemas electorales de origen diferente, pero ambos revisados por el Tribunal Constitucional. Para la Cámara de Diputados, tras la decisión de 2017, fue aplicado el sistema Italicum, que preveía la asignación del 55\% de escaños a la lista que hubiera obtenido el $40 \%$ de votos. En caso de que esto no hubiese ocurrido, habría tenido lugar una repartición proporcional entre las fuerzas que hubieran superado el umbral mínimo de votos. Para el Senado, en cambio, seguía siendo aplicable el llamado "Porcellum" corregido por la Consulta (por eso llamado Consultellum), que preveía una repartición proporcional de los escaños asignados a las regiones entre los partidos políticos (listas y coaliciones) que hubieran superado los umbrales mínimos de votos (siempre regionales). Ambos sistemas habrían tenido un efecto proporcional frente a la actual situación política italiana (que hace irrealizable para las listas la obtención del $40 \%$ de votos). Los partidos, impulsados también por las declaraciones del Presidente de la República ${ }^{31}$,

29 De Lungo, D. (2017). "Il Premio di maggioranza alla lista, fra governabilità e legittimità costituzionale. Considerazioni (anche) a valle della sentenza n. 35 del 2017”. Rivista AIC, 2, pp. 8-10. En contra de la opinión del Tribunal, véase Pasquino, G. (2017). "La Corte decide di decidere ma non coglie la natura del ballottaggio”. Forum di Quaderni Costituzionali, 6 de abril.

30 Para un análisis del sorteo en derecho público, véase Zei, A. (2017). "L'arbitrato del caso: applicazioni del metodo del sorteggio nel Diritto pubblico”. Nomos, n. 1.

31 Cabe recordar el discurso de fin del año del Presidente de la República Mattarella, que deseaba dos sistemas homogéneos entre Cámara y Senado. En abril de 2017 afirmó lo urgente que era aprobar una nueva ley electoral. 
empezaron a debatir para tratar de llegar a un acuerdo sobre un nuevo sistema electoral homogéneo para Cámara de Diputados y Senado.

El 26 de octubre de 2017, el Senado aprobó de manera definitiva la ley 165/2017 sobre la revisión del sistema de elección de Cámara y Senado (el así llamado Rosatellum $)^{32}$. El modelo electoral escogido es el modelo mixto, que prevé la asignación del $36 \%$ (aproximadamente) de escaños con un sistema mayoritario (circunscripciones uninominales con modelo plurality) y del 64\% (aproximadamente) de escaños con un sistema proporcional. Cada partido o coalición presentará (además del candidato "uninominal") una lista de candidatos (no menos de dos y no más de cuatro) en las 232 circunscripciones de la Cámara y en las 116 circunscripciones del Senado. El sistema no prevé preferencias, pero hay unas listas cortas y bloqueadas, siguiendo las indicaciones que el Tribunal dio con la decisión 1/2014. Los candidatos pueden presentarse en más de una lista plurinominal (con un máximo de cinco), pero en una sola circunscripción uninominal.

La ley electoral prevé un umbral mínimo de votos del 3\% sobre base nacional (tanto para el Senado como para la Cámara); mientras que, para las coaliciones, el umbral mínimo de votos se eleva al $10 \%$. Una importante novedad del Rosatellum es la predicción de un umbral mínimo para el uso de los votos en coalición, que puede sumar sólo los votos de los partidos que superan el 1\%. Bajo este umbral, en cambio, los votos no son computados.

Finalmente, hay que subrayar que este sistema no prevé el voto disjunto, aquel voto que el candidato recibe mayoritariamente y que se añadirá también al partido/ coalición en la sección proporcional. Debido a esta característica este sistema electoral puede ser clasificado como "mixto con combinación y voto fusionado" 33 , con partes mayoritaria y proporcional desunidas a nivel funcional y mecánico, pero sobrepuestas territorialmente, y sin posibilidad alguna de expresar dos votos diferentes para cada una de ellas ${ }^{34}$. El voto para el candidato uninominal, de hecho, tiene como objetivo el de personalizar el voto en la lista o, mejor dicho, de identificar a una personalidad política de referencia del partido o de la coalición en ámbito local. En resumen, la cuota mayoritaria sirve para permitir al partido encarrilar los votos hacia el candidato más importante en ámbito territorial, que entonces acaba por configurarse como cabeza de lista con respecto de las candidaturas proporcionales, en caso de listas coligadas, como candidato único propuesto por parte de toda la coalición en ámbito local ${ }^{35}$.

32 Para una análisis comltea, Armanno, M. (2018). Personale, Uguale, Libero e Segreto, Nápoles, Editoriale Scientifica Italiana, pp. 251- 284.

33 Véase Chiaramonte, A. (1998). "I sistemi elettorali misti: una classificazione". Rivista Italiana di Scienza Politica, 2, p. 239.

34 Crítico en este punto Caravita, B. (2017). "Sul disegno di legge in materia elettorale". Federalismi, 20, p.5.

35 Se ha hablado de un «súper-cabeza de lista válido para cada una de las listas coligadas», véase Fusaro, C. (2017). Audizione Senato 19 ottobre 2017, en http://www.senato.it/application/xmanager/projects/.../ PROF._FUSARO.pdf 


\section{LAS ELECCIONES DEL 4 DE MARZO DE 2018 Y EL ESTANCAMIENTO EN LA FORMACIÓN DEL GOBIERNO}

Si las elecciones italianas de 2013 fueron definidas un "terremoto con liquefazione" 36 («terremoto con licuefacción»), las de 2018 pueden ser acercadas a una deflagración del sistema. Como era previsible, el sistema electoral ha tenido efectos proporcionales. El Parlamento italiano se ha dividido en tres agrupaciones. El Movimento 5 Stelle, una fuerza política de formación reciente, traedora de instancias antisistema guiada por Luigi Di Maio, el primer partido con casi el 33\% de votos.

Otra agrupación, representada por la coalición de centroderecha, que obtuvo en total el $37 \%$ de votos, está compuesta por tres partidos: Lega, Forza Italia y Fratelli d'Italia. La primera obtuvo aproximadamente el $18 \%$ de votos, presionando sobre temáticas antieuropeístas y antiinmigración y está guiada por Matteo Salvini, que se ha convertido también en el nuevo líder de la coalición, frente al resultado electoral. Forza Italia, que sigue teniendo a Silvio Berlusconi como representante, obtuvo sólo el $14 \%$ de votos. Este resultado es de extrema importancia. Por primera vez en la historia de la agrupación italiana de centroderecha, el partido dirigido por Silvio Berlusconi no fue la fuerza más votada de la coalición, con una deriva populista de toda la coalición. Fratelli d'Italia, un partido conservador y nacionalista, obtuvo el $4 \%$.

Por último, el Partito Democratico (centroizquierda), que ha dirigido Italia en los 5 años previos a las elecciones de 2018, fue el gran derrotado, puesto que obtuvo alrededor del $19 \%$ de votos. Inmediatamente después de este resultado, Matteo Renzi dimitió como secretario del partido.

De tal maneraEntonces, el Parlamento italiano se presenta tripartido con la imposibilidad para las fuerzas políticas de obtener, de manera autónoma, la mayoría necesaria para gobernar ${ }^{37}$.

La legislatura empezó el 23 de marzo de 2018 y tuvo que hacer frente a la elección de los presidentes de las dos Cámaras, que fueron elegidos tras un breve estancamiento y gracias a un acuerdo entre la centroderecha y el Movimento 5 Stelle. Éste eligió, por primera vez, uno de sus representantes en la cumbre del Estado. Se trata de Roberto Fico. Para el Senado de la República, en cambio, fue elegida presidenta Maria Elisabetta Alberti Casellati, exponente de centroderecha (representando a Forza Italia).

Inmediatamente después empezó el procedimiento para la formación del nuevo Gobierno. El Presidente de la República, Mattarella, consultó tres veces a las fuerzas políticas sin que ello conllevara a formar un Gobierno. En la crisis que hubo durante la “formación del Gobierno", que ocurrió de marzo a mayo de 2018, se desarrolló una

36 El 17 de abril de 2013 el Profesor Lanchester organizó en la Universidad "La Sapienza” de Roma una conferencia con este título, ahora disponible en Radio Radicale.

37 Está presente en el Parlamento también "Liberi e Uguali”, una fuerza de izquierda encabezada por el ex Presidente del Senado, Pietro Grasso. Obtuvo poco más del 3\% de votos. 
situación inédita para la historia republicana: un doble mandato exploratorio con una fuerte connotación política. De hecho, Mattarella entregó un mandato exploratorio “vinculado". El Jefe del Estado primero encomendó a la Presidenta del Senado, Maria Elisabetta Alberti Casellati, «la tarea de verificar la existencia de una mayoría parlamentaria entre los partidos de la Coalición de centroderecha y el Movimento 5 Stelle y de una indicación compartida sobre el otorgamiento del encargo de Presidente del Gobierno con el fin de constituir el Gobierno» ${ }^{38}$. Tras dos días de consultas, la presidenta informó sobre los éxitos de este diálogo. Según la misma: «no obstante la diversidad de opiniones presentes, se permitió poner en evidencia motivos de reflexión política» ${ }^{39}$. Las tensiones entre la coalición de centroderecha y el Movimento 5 Stelle se debían a la presencia de Forza Italia (y sobre todo de Berlusconi) en una eventual alianza de gobierno.

A los pocos días, el Jefe del Estado convocó al Presidente de la Cámara, Roberto Fico, a quien «encomendó la tarea de verificar la posibilidad de una alianza de mayoría parlamentaria entre el Movimento 5 Stelle y el Partito Democratico para constituir el Gobierno» ${ }^{40}$.

Los “exploradores" sonsacaron aspectos positivos en su propia intervención, pero las reales mejoras en la situación política se revelaron fatuas, puesto que el pasado 7 de mayo el Presidente de la República convocó nuevas (y breves) consultas. Tras esas consultas, Mattarella afirmó que: después de una evaluación «cuidadosa y puntual de todas las soluciones posibles en un Parlamento marcado, como se sabe, por tres fuerzas predominantes, ninguna de las cuales posee la mayoría. Una condición, ésta, que necesita que dos entre ellas lleguen a un acuerdo para gobernar» ${ }^{41}$. Frente a esta situación, el Jefe del Estado adelantó el nombramiento de «un gobierno neutral, de servicio. [...] Un gobierno neutral en comparación con las fuerzas políticas». Por supuesto, el Presidente Mattarella había deseado la realización de una alianza política para dar vida a un nuevo gobierno con plenitud de funciones. Después de pocos días, el Movimento 5 Stelle y la Lega comunicaron su voluntad de enfrentarse sobre la posibilidad de formar un gobierno. El 24 de mayo el Presidente Mattarella dio el encargo de formar el Gobierno al profesor Conte, indicado por Di Maio y Salvini para ejecutar el "contracto de gobierno" que ellos mismos habían firmado ${ }^{42}$. Esta tentativa fracasó por diferencias sobre el nombramiento del Ministro de Economía, por causa del veto del Presidente Mattarella a Paolo Savona, un profesor euroescéptico ${ }^{43}$. El Presidente de la República convocó a Cottarelli para formar un gobierno neutral ${ }^{44}$,

38 Comunicado del Quirinal, 18 de abril de 2018.

39 Declaración de la Presidenta del Senado, Maria Elisabetta Alberti Casellati, Palacio del Quirinal, 20 de abril de 2018.

${ }^{40}$ Comunicado del Quirinal, 23 de abril de 2018.

41 Comunicado del Quirinal, 7 de mayo de 2018.

42 Comunicado del Quirinal, 23 de mayo de 2018.

43 Comunicado del Quirinal, 27 de mayo de 2018.

44 Comunicado del Quirinal, 28 de mayo de 2018. 
pero tras unos días, la Lega y el Movimento 5 Stelle decidieron formar otra vez el Gobierno, aceptando las reservas de Mattarella sobre Savona, desviado al Ministerio de Asuntos Europeos.

Finalmente, el 1 de junio el Gobierno Conte ocupó su puesto ${ }^{45}$ y el 5 y 6 de junio ganó la confianza de las dos Cámaras. La crisis de gobierno más larga de la historia terminó de esta manera.

\section{UN SISTEMA EN LA ANSIADA BÚSQUEDA DE UN EQUILIBRIO}

El nuevo sistema electoral ha sido comparado a una "ley-puente" 46 . La situación política italiana dificultó una elección mayoritaria más estable ${ }^{47}$. Es evidente que los partidos políticos tradicionales, en una situación de incertidumbre, optaron por un sistema con efectos proporcionales para evitar la irrelevancia en los próximos años y para resultar relevantes para la formación de la mayoría gubernamental en la esta legislatura, en la que serán decididas las coaliciones postelectorales. La larga crisis de gobierno que hubo después de las elecciones del 4 de marzo fue hija de la incapacidad de los partidos para relacionarse con la nueva dinámica proporcional, tras veinte años de sistema mayoritario.

Durante los meses de agosto y septiembre de 2019, se produjo otra crisis de gobierno que llevó a la Liga a situarse en la oposición, con la consiguiente formación de un gobierno de coalición compuesto por el Movimento 5 Stelle, el Partito Democratico y el partido de izquierda Liberi e Uguali ${ }^{48}$. Tras este acontecimiento, la cuestión sobre la ley electoral ha vuelto a recobrar importancia. Ahora, la mayoría aboga por un sistema totalmente proporcional (con umbrales mínimos de voto), mientras que la Lega aboga por un sistema totalmente mayoritario. Por esta razón, a finales de septiembre, 7 Consejos regionales ${ }^{49}$ pidieron un referéndum abrogativo (ex art. 75 Const. $^{50}$ ) para abolir la parte proporcional de la ley electoral n. 165/2017. La decisión

45 Comunicado del Quirinal, 31 de mayo y 1 de junio de 2018.

46 Ceccanti, S. (2017). "Legislazione elettorale. Italia. Una nuova legge-ponte nella transizione che prosegue". www.dpce.it.

47 Ortona, G., Ottone, S. y Ponzano, F. (2007). "A simulative assessment of the Italian electoral system”, en Padovano F. y Ricciuti, R., Italian Institutional Reforms: A Public Choice Perspective, Nueva York, Springer, pp. 21-36.

48 Algunas semanas después, Matteo Renzi abandonó el Partido Democrático, formando un nuevo partido llamado Italia Viva, que apoya asimismo al gobierno.

${ }^{49}$ Los consejos regionales de Cerdeña, Lombardía, Friuli, Piamonte, Abruzos, Liguria y Basilicata han hecho la propuesta. El Véneto, que presentó la propuesta, no completó el procedimiento.

50 Art. 75 Const.: «Se convocará referéndum popular para decidir sobre la derogación total o parcial, de una Ley o de un acto con fuerza de Ley, cuando lo soliciten quinientos mil electores o cinco Consejos regionales. No se admitirá el referéndum para las leyes tributarias y presupuestarias, de amnistía y de indulto, ni de autorización para ratificar tratados internacionales. Tendrán derecho a participar en el referéndum todos los ciudadanos llamados a elegir la Cámara de Diputados. La propuesta sometida a referéndum será aprobada si ha participado en su votación la mayoría de quienes tengan derecho de 
de admitir este referéndum pesa sobre el Tribunal Constitucional que, previsiblemente, en el próximo mes de enero 2020, se pronunciará sobre el asunto ${ }^{51}$. Ante tales circunstancias, será necesario modificar la ley electoral para hacer frente a la última reforma constitucional, aprobada el pasado mes de octubre ${ }^{52}$, que debería reducir a 400 el número de los miembros de la Cámara de Diputados y a 200 el de los senadores (en el supuesto de que se celebre referéndum ex artículo 138 Const., esa consulta ha de arrojar un resultado positivo y confirmatorio ${ }^{53}$ ). Quizás estas simples revisiones técnicas puedan despertar controversias entre la mayoría y la oposición sobre las características esenciales de la legislación electoral.

Sería de esperar que esta nueva ley electoral pueda durar el mayor tiempo posible ${ }^{54}$ o que se debería cambiar de inmediato por una más duradera, pero la visión optimista choca con la realidad. Después de un largo período en el que se han experimentado sistemas mayoritarios (relacionados con la alternancia de gobierno), Italia vuelve hacia una democracia consensual ${ }^{55}$, en la que jugarán un papel fundamental la predisposición al compromiso y la presencia de coaliciones postelectorales. No significa que si el sistema político italiano tuviera que estabilizarse de nuevo hacia un modelo bipartidista, no se decida virar otra vez hacia una ley mayoritaria. Es probable que la presencia de una agrupación atípica, como la del Movimento 5 Stelle, haya reforzado la idea de una ley electoral de transición para impedir la victoria del partido de Grillo.

El resultado electoral del 4 de marzo y las dificultades en la formación de un gobierno convencieron a unas fuerzas políticas para que cambiaran otra vez la ley electoral, pero sin proporcionar elementos que permitieran desarrollar el razonamiento. El verdadero problema de la legislación electoral italiana es que se esboza basán-

voto y si se alcanza la mayoría de los votos válidamente emitidos. La Ley determinará las modalidades de celebración del referéndum».

51 Parte de la doctrina cree que no habrá alguna admisión del referéndum, vèase Di Chiara, A. (2019). "Dal 'Rosatellum' al maggioritario puro via referendum? Un quesito difficilmente ammissibile”. www. lacostituzione.info, 29 septiembre

52 Texto de ley constitucional «Modifiche agli articoli 56, 57 e 59 della Costituzione in materia di riduzione del numero dei parlamentari» publicado en la Gazzetta Ufficiale, 12 octubre 2019, n. 240.

53 Art. 138 Const.: «Las leyes de revisión de la Constitución y demás leyes constitucionales serán adoptadas por cada una de las Cámaras en dos votaciones sucesivas con intervalo de espera entre ellas de no menos de tres meses, y serán aprobadas por mayoría absoluta de los miembros de cada Cámara en la segunda votación. Dichas leyes serán sometidas a referéndum popular, dentro de los tres meses siguientes a su publicación, cuando así lo solicite una quinta parte de los miembros de una Cámara o quinientos mil electores o cinco Consejos Regionales. La Ley sometida a referéndum no se promulgará si no fuere aprobada por la mayoría de los votos válidos. No se convocará referéndum si la Ley se aprobara en la segunda votación por cada una de las Cámaras por mayoría de dos tercios de sus respectivos componentes».

${ }^{54}$ Lo desea Lupo, N. (2017). "Verso un'auspicabile stabilizzazione della legislazione elettorale italiana. Alcuni spunti sulla legge n. 165 del 2017, in rapporto alla Costituzione”. Federalismi, 22, pp.38-41.

55 Término utilizado por Lijphart, A. (1968). "Typologies of democratic systems”. Comparative Political Studies, 1, pp.3-44.

N. ${ }^{\circ} 106$, septiembre-diciembre 2019, págs 265-283 
dose en los sondeos presentes durante su redacción y para garantizar la mayoría en servicio o para evitar la victoria de una fuerza de oposición ${ }^{56}$. Los partidos no tienen el coraje de hacer elecciones de gran envergadura e imaginar "reglas de juego" que puedan ser válidas durante mucho tiempo.

La estabilización del sistema electoral italiano parece muy lejana y no se da por descontado que en una situación fluida no vuelva a ser protagonista también el Tribunal Constitucional.

Title:

The Italian electoral system: what the legislator does, the Court undoes

\section{Summary:}

1. Premise - 2. From 2005 to 2014: three elections with an unconstitutional electoral law - 3. A new electoral law never applied, but unconstitutional -4 . An approved electoral law (in the run-up to the elections) that displeases everyone. - 5. The elections of March 4, 2018 and the deadlock in the formation of the government - 6. A system searching for a coveted balance.

\section{Resumen}

En este escrito se analiza el controvertido régimen electoral de Italia en los últimos cinco años. Después de la importante decisión 1 de 2014 de la Corte costituzionale, el sistema electoral italiano se encontró entre los más inestables, al declararse inconstitucional dos veces la ley electoral. Las últimas elecciones se han llevado a cabo a través de un sistema mixto que ha provocado un estancamiento en la formación del gobierno. La nueva ley electoral ha sido objeto de crítica desde diferentes sectores y parece ser transitoria, en espera de un nuevo y codiciado equilibrio político.

\section{Abstract}

This paper's aim is to reconstruct the electoral question that has affected Italy for four years. After the famous judgement n.1/2014 of the Consti-

56 Para una lectura de la historia electoral italiana, véase Passarelli, G. (2018). "Electoral systems in Context: Italy", en Herron, E. S., Pekkanen R. J. y Shugart M. S., The Oxford Handbook of Electoral Systems, Oxford, Oxford Press, pp. 851-870. Clementi, F. (2015). "Vent'anni di legislazione elettorale (1993- 2013). Tra il già e il non ancora”. Rivista Trimestrale di diritto pubblico, 2, pp. 557- 609. 
tutional Court, the Italian electoral political system has been one of the most unstable, considering that another electoral system was declared unconstitutional. The latest elections were held with a mixed system that caused a deadlock in the formation of the government. The new electoral law has dissatisfied everyone and seems to be temporary, waiting for a new and coveted political balance.

\section{Palabras clave:}

Ley electoral; Tribunal Constitucional; elecciones; Italia

\section{Keywords:}

Electoral Law; Constitutional Court; Elections; Italy 